\title{
NUMBERS OF SOLUTIONS OF CONGRUENCES: POINCARÉ SERIES FOR STRONGLY NONDEGENERATE FORMS
}

\begin{abstract}
JAY R. GOLDMAN ${ }^{1}$
ABstract. Let $p$ be a fixed prime, $f\left(x_{1} \ldots \ldots x_{h}\right)$ a polynomial over $\mathbf{Z}_{p}$, the $p$-adic integers, $c_{n}$ the number of solutions of $f=0$ over $\mathbf{Z} / p^{\prime \prime} \mathbf{Z}$ and $P_{f}(t)=\sum_{t \cdot{ }_{0} c t^{\prime} \text { the }}$ Poincare series. A general approach to computing $c_{n}$ and $P_{f}(t)$ is given and explicit formulas are derived for strongly nondegenerate forms
\end{abstract}

1. Introduction. Let $p$ be a fixed prime and $f(x)=f\left(x_{1} \ldots \ldots x_{k}\right)$ a polynomial with coefficients in $\mathbf{Z}_{p}$, the $p$-adic integers. Let $c_{n}$ denote the number of solutions of $f=0$ over the ring $\mathbf{Z} / p^{n} \mathbf{Z}$, with $c_{0}=1$. Then the Poincare series $P_{f}(t)$ is the generating function

$$
P_{f}(t)=\sum_{i=0}^{\infty} c_{i} t^{\prime}
$$

This series was introduced by Borevich and Shafarevich [1, p. 47] and is the analogue for the rings $\mathbf{Z} / p^{n} \mathbf{Z}$ of the zeta function of $f$ over finite fields.

Borevich and Shafarevich conjectured that $P_{f}(t)$ is a rational function of $t$ for all polynomials. This was proved by Igusa in 1975, in a more general setting, using a mixture of analytic and algebraic methods in which a key tool is the resolution of singularities of algebraic varieties $[2,3]$. The proof is nonconstructive and further studies of the problem by Igusa are summarized in his Tata Notes [4]. D. Meuser [6] extended Igusa's theorem from hypersurfaces to algebraic varieties. E. Stevenson [7] treated polynomials of the form $\Sigma x_{i}^{d_{1}}$ where $p \nmid d_{1}$, using Jacobi sums. Her methods. which are fully constructive, are quite complicated for carrying out computations.

In this paper an elementary algebraic-combinatorial approach is given for deriving a recursion for $c_{n}$ and computing the Poincare series for strongly nondegenerate forms. In future papers I will present an analogous approach to polynomials in one variable and classes of algebraic curves. In \$2 some general remarks and a lemma which is fundamental for counting solutions are presented. In $\$ 3$ the results are derived for strongly nondegenerate forms.

I want to thank Steve Sperber for telling me about the problem of Poincaré series and for many valuable discussions and James M. Vaughn, Jr. and the Vaughn Foundation Fund for their support of my studies in number theory.

Received by the editors May 14, 1982.

1980 Mathematics Subject Classification. Primary 12C99, 50A15, 10C10, 10C03.

'The work was supported in part by the Vaughn Foundation Fund and NSF Grant MCS 77-28421. 
2. Fundamental Lemma. A solution of $f(x)=0$ over $\mathbf{Z} / p^{n} \mathbf{Z}$ can be represented as a $k$-tuple $x=\left(x_{i}, \ldots, x_{k}\right), 0 \leqslant x_{i} \leqslant p^{n}-1$, where we also think of each $x_{i}$ with its unique expansion in base $p$ :

$$
x_{i}=\sum_{j=0}^{n-1} a_{j}^{(i)} p^{j}, \quad 0 \leqslant a_{j}^{(i)} \leqslant p-1 .
$$

Since $f(x) \equiv 0\left(\bmod p^{n}\right)$ implies that $f(x) \equiv 0\left(\bmod p^{l}\right)$ for all $l<n$ we also have that every such $x$ is congruent to a unique $x^{\prime}$ in $\left(\mathbf{Z} / p^{\prime} \mathbf{Z}\right)^{k} \bmod p^{\prime}$. In a base $p$ expansion each entry in $x^{\prime}$ consists of the first $l-1$ terms of the corresponding entry in $x$. We say that $x$ is a descendant of $x^{\prime}$.

The fact that each solution $\left(x_{1}, \ldots, x_{k}\right) \bmod p^{n}$ can branch to a finite number of descendants $\bmod p^{n+1}$, all of the form $\left(\ldots, x_{t}+b_{i} p^{n}, \ldots\right)$, is the basis of our counting arguments.

Definition. A solution $a=\left(a_{1}, \ldots, a_{k}\right)$ in $(\mathbf{Z} / p \mathbf{Z})^{k}$ of $f \equiv 0(\bmod p)$ is nonsingular if the partial derivatives $f_{x_{1}}(a)$ are not all $\equiv 0(\bmod p)$; otherwise the solution is singular.

A basic step in our counting arguments is provided by the following simple application of Taylor's theorem.

Fundamental Lemma. Let $f\left(x_{1}, \ldots, x_{k}\right)$ be a polynomial over $\mathbf{Z}_{p}$ and let $a=$ $\left(a_{1}, \ldots, a_{k}\right)$ in $(\mathbf{Z} / p \mathbf{Z})^{k}$ be a nonsingular solution of $f \equiv 0(\bmod p)$. Then there are $p^{(n-1)(k-1)}$ solutions of

$$
f \equiv 0 \quad\left(\bmod p^{n}\right)
$$

i.e. solutions in $\left(\mathbf{Z} / p^{n} \mathbf{Z}\right)^{k}$, descending from $a$.

Proof. Let $a^{(n)}=\left(a_{1}^{(n)}, \ldots, a_{h}^{(n)}\right)$ be a solution of (1) descending from $a=a^{(1)}$. We shall prove that there are $p^{k-1}$ solutions $a^{(n+1)}$, of $f \equiv 0\left(\bmod p^{n+1}\right)$, descending from $a^{(n)}$. The lemma then follows by a simple induction.

Thinking of our numbers as expanded in the base $p$ we must decide for which $b_{1}$. $0 \leqslant b_{1} \leqslant p-1$, the vector $a^{(n+1)}=\left(a_{1}^{(n)}+b_{1} p^{n}, \ldots, a_{h}^{(n)}+b_{k} p^{n}\right)$ will be a solution of $f \equiv 0\left(\bmod p^{n+1}\right)$. By Taylor's theorem

$$
f\left(a^{(n)}+b p^{n}\right)=f\left(a^{(n)}\right)+\sum_{1} f_{x_{1}}\left(a^{(n)}\right) b_{1} p^{n}+\text { higher order terms. }
$$

Now $f\left(a^{(n)}\right) \equiv 0\left(\bmod p^{n}\right)$ and the higher order terms contain $p$ to a power larger than $n$. Therefore, recalling that $a_{i}^{(n)} \equiv a_{i}^{(1)}(\bmod p)$ and dividing both sides of $(2)$ by $p^{n}$, we have

$$
f\left(a^{(n+1)}\right) \equiv 0 \quad\left(\bmod p^{n+1}\right)
$$

if and only if

$$
c+\sum_{1} f_{x_{1}}\left(a^{(1)}\right) b_{1} \equiv 0 \quad(\bmod p)
$$

But by assumption $f_{x_{i}}\left(a^{(1)}\right) \neq 0$ for some $i$, say $i=l$. Therefore for any choice of the $b_{1}, i \neq l$, we can solve for $b_{l}(\bmod p)$. Since there are $p$ choices for each $b_{1}$. there are $p^{k-1}$ solutions of $f \equiv 0\left(\bmod p^{n+1}\right)$ descending from $a^{(n)}$. Q.E.D. 
Since a solution of $f \equiv 0\left(\bmod p^{n}\right)$ "branches" to its descendants $\bmod p^{n+1}$ it can be very useful to interpret our problems using the combinatorial structure of rooted trees. If $a$ is a solution of $f=0$ in $(Z / p Z)^{k}$, then we let $a$ be the rooted vertex of a tree and take all its descendants as the other vertices. Every solution mod $p^{n}$ is connected by edges oriented outward to each of its descendants mod $p^{n+1}$.

Proposition. If $a$ is a solution of $f \equiv 0(\bmod p)$ which has infinitely many descendants then there is a solution $\bar{a}$ in $\mathbf{Z}_{p}^{k}$ of $f=0$ such that $\bar{a} \equiv a(\bmod p)$.

Proof. The tree $T$ associated to $a$, as described above, is an infinite tree by assumption. However each vertex of $T$ is incident to only a finite number of vertices since there are only a finite number of solutions of $f \equiv 0\left(\bmod p^{n}\right)$ for each $n$. Therefore, by König's lemma [5, p. 38] there exists an infinite directed branch beginning at $a$ with vertices $a=a^{(1)}, a^{(2)} \ldots$ which satisfy $f\left(a^{(n)}\right) \equiv 0\left(\bmod p^{n}\right)$ and $a^{(n+1)} \equiv a^{(n)}\left(\bmod p^{n}\right)$. Hence there exist integers $\left\{c_{i}^{(\jmath)}, j=1, \ldots, k, i=\right.$ $0,1, \ldots\}$, with $0 \leqslant c_{i}^{(\jmath)} \leqslant p-1$, such that

$$
a^{(n)}=\left(\sum_{i=0}^{n-1} c_{i}^{(1)} p^{1} \ldots . \sum_{i=0}^{n-1} c_{i}^{(h)} p^{l}\right) .
$$

Let $\bar{a}=\left(\sum_{l=0}^{\infty} c_{l}^{(1)} p^{\prime} \ldots, \sum_{l=0}^{\infty} c_{1}^{(k)} p^{\prime}\right)$. Then $\bar{a}$ is in $\mathbf{Z}_{p}^{h}$ and by the continuity of $f$. $f(\bar{a})=0$. Clearly $\bar{a} \equiv a(\bmod p)$.

A well-known result [1, p. 43] which follows immediately is

COROLlaRY. If $a$ is a nonsingular solution of $f \equiv 0(\bmod p)$, then there exists an $\bar{a}$ in $\mathbf{Z}_{p}^{h}$ such that $f(\bar{a})=0$ and $\bar{a} \equiv a(\bmod p)$.

Proof. By the fundamental lemma $a$ has infinitely many descendants and the Proposition applies.

3. Strongly nondegenerate forms. By a strongly nondegenerate form we mean a homogeneous polynomial $F\left(x_{1}, \ldots, x_{h}\right)$ such that the only singular solution of $F \equiv 0$ $(\bmod p)$ is $(0,0, \ldots, 0)$. Examples of such forms include $\sum_{i=1}^{h} e_{i} x_{i}^{d}$, where $p \nmid d$ and the $e_{1}$ are $p$-adic units, and $x^{2}+y^{2}+x y$ where $p \neq 2,3$.

THEOREM. Let $F\left(x_{1}, \ldots, x_{h}\right)$ be a strongly nondegenerate form of degree $d$ with coefficients in $\mathbf{Z}_{p}$. Let $c_{n}$ be the number of solutions of $F=0$ in $\left(\mathbf{Z} / p^{n} \mathbf{Z}\right)^{h}$, with $c_{0}=1$. Then

(A) we have

$$
c_{n}=\left(c_{1}-1\right) p^{(n-1)(h-1)}+p^{h(n-1)}
$$

for $1 \leqslant n \leqslant d$,

$$
c_{n}=\left(c_{1}-1\right) p^{(n-1)(h-1)}+p^{h(d-1)} c_{n \cdot d}
$$

for $d<n$.

(B) The Poincaré series

$$
P_{F}(t)=\frac{R(t)}{\left(1-p^{h-1} t\right)\left(1-p^{h(d-1)} t^{d}\right)}
$$

where $R(t)$ is a polynomial of degree $d$, which is effectively, and easily, computable. 
Igusa [4, p. 104] gave a different derivation for the denominator of $P_{F}(t)$.

Proof. To derive the recursions in (A) let $c_{n}=N_{n}+S_{n}$ where $N_{n}$ denotes the number of solutions mod $p^{n}$ descending from nonsingular solutions $\bmod p$ and $S_{n}$ denotes the number of solutions mod $p^{n}$ descending from $(0, \ldots, 0)$, the only singular solution $\bmod p$.

Since $(0, \ldots, 0)$ is the only singular solution $\bmod p$ there are $c_{1}-1$ nonsingular solutions $\bmod p$ and by the fundamental lemma

$$
N_{n}=\left(c_{1}-1\right) p^{(n-1)(k-1)} .
$$

To compute $S_{n}$, we work by cases.

(i) $n=1$.

By definition $S_{n}=1$.

(ii) $2 \leqslant n \leqslant d$.

Let

$$
a=\left(\sum_{i=1}^{n-1} a_{i}^{(1)} p^{i}, \ldots, \sum_{i=1}^{n-1} a_{i}^{(k)} p^{i}\right)
$$

$0 \leqslant a_{i}^{(\prime)} \leqslant p-1$, be an arbitrary vector $\equiv 0(\bmod p)$. Then $a=p a^{*}$ where $a^{*}=$ $\left(\sum_{i=1}^{n-1} a_{i}^{(1)} p^{i-1}, \ldots, \sum_{i=1}^{n-1} a_{i}^{(k)} p^{i-1}\right)$. Since $d \geqslant n$, we have, by the homogeneity of $F$,

$$
F(a)=F\left(p a^{*}\right)=p^{d} F\left(a^{*}\right) \equiv 0 \quad\left(\bmod p^{n}\right) .
$$

Thus all such vectors are solutions and since there are $k(n-1)$ coefficients $a_{i}^{(\prime)}$ and $p$ choices for each coefficient we have $S_{n}=p^{k(n-1)}$.

(iii) $d<n$.

Using the notation of case (ii)

$$
F(a)=p^{d} F\left(a^{*}\right) \equiv 0 \quad\left(\bmod p^{n}\right)
$$

if and only if $F\left(a^{*}\right) \equiv 0\left(\bmod p^{n-d}\right)$.

But $p^{n-d}, p^{n-d+1}, \ldots, p^{n-2}$ are $\equiv 0\left(\bmod p^{n-d}\right)$ and we have free choice for each of their $d-1$ coefficients in each term of $a^{*}$ yielding $p^{k(d-1)}$ choices. We want the remaining part of $a^{*}$, viz. $\left(\sum_{i=1}^{n-d-1} a_{i}^{(1)} p^{j-1}, \ldots, \sum_{i=1}^{n-d-1} a_{i}^{(k)} p^{j-1}\right)$ to be an arbitrary solution of $F \equiv 0\left(\bmod p^{n-d}\right)$ and there are exactly $c_{n-d}$ such solutions.

Hence $S_{n}=p^{k(d-1)} c_{n-d}$ and part (A) is proved.

To compute $P_{F}(t)$ we form a generating function using equations (3) and (4). Note that, since $c_{0}=1,(3)$ and (4) agree for $n=d$. Thus we have

$$
\sum_{n=d}^{\infty} c_{n} t^{n}=\left(c_{1}-1\right) t \sum_{n=d}^{\infty} p^{(n-1)(k-1)} t^{n-1}+p^{k(d-1)} t^{d} \sum_{n=d}^{\infty} c_{n-d} t^{n-d}
$$

or

$$
P_{F}(t)-\sum_{n=0}^{d-1} c_{n} t^{n}=\frac{\left(c_{1}-1\right) t^{n}\left(p^{(k-1)} t\right)^{d-1}}{\left(1-p^{k-1} t\right)}+p^{k(d-1)} t^{d} P_{F}(t)
$$

Thus letting $Q_{F}(t)=\sum_{n=0}^{d-1} c_{n} t^{n}$ we have

$$
P_{F}(t)=\frac{\left(c_{1}-1\right) p^{(k-1)(d-1)} t^{d}+\left(1-p^{k-1} t\right) Q_{F}(t)}{\left(1-p^{k-1} t\right)\left(1-p^{k(d-1)} t^{d}\right)}
$$


where $Q_{F}(t)$ is a polynomial of degree $d-1$ which can be computed directly from equation (3) by summing geometric series. By looking at the expression for $c_{d-1}$ in (3) we see that the numerator of $P_{F}(t)$ is a polynomial of degree $d$.

\section{REFERENCES}

I. Z. I. Borevich and I. R. Shafarevich. Number theon, Academic Press. New York. 1966

2. J. Igusa. Complex powers and astmptotic expansions. II. J. Reine Angew: Math $278 / 279$ (1975). $307-321$

3. Some obsercations on higher degree characters. Amer. J. Math. 99 (1977), 393-417.

4. Lectures on forms of higher degree. Tata Institute Notes, Springer-Verlag. Berlin and Neu York, 1978

5. D. Knuth. The art of computer programming. Vol. 1: Fundamental algorithms, 2nd ed., Addison-Wesley, Reading. Mass., 1973.

6. D. Meuser, On the rationality of certain generating functions. Math. Ann. 256 (1981), 303-310.

7. E. Stevenson. The rationality of the Poincare series of a diagonal form. Acta Arith. (to appear).

Department of Mathematics, University of Minnesota. Minnfapolis. Minnesota 55455 